\title{
Horizon quantum mechanics of rotating black holes
}

\author{
Roberto Casadio $^{1,2, a}$, Andrea Giugno ${ }^{3, b}$, Andrea Giusti ${ }^{1,2,3, c}$, Octavian Micu ${ }^{4, d}$ \\ ${ }^{1}$ Dipartimento di Fisica e Astronomia, Università di Bologna, via Irnerio 46, 40126 Bologna, Italy \\ 2 I.N.F.N., Sezione di Bologna, I.S. FLAG, via B. Pichat 6/2, 40127 Bologna, Italy \\ ${ }^{3}$ Arnold Sommerfeld Center, Ludwig-Maximilians-Universität, Theresienstraße 37, 80333 Munich, Germany \\ ${ }^{4}$ Institute of Space Science, Bucharest, P.O. Box MG-23, 077125 Bucharest-Magurele, Romania
}

Received: 30 January 2017 / Accepted: 4 May 2017 / Published online: 18 May 2017

(C) The Author(s) 2017. This article is an open access publication

\begin{abstract}
The horizon quantum mechanics is an approach that was previously introduced in order to analyze the gravitational radius of spherically symmetric systems and compute the probability that a given quantum state is a black hole. In this work, we first extend the formalism to general spacetimes with asymptotic (ADM) mass and angular momentum. We then apply the extended horizon quantum mechanics to a harmonic model of rotating corpuscular black holes. We find that simple configurations of this model naturally suppress the appearance of the inner horizon and seem to disfavor extremal (macroscopic) geometries.
\end{abstract}

\section{Introduction}

Astrophysical compact objects are known to be usually rotating, and one correspondingly expects most black holes formed by the gravitational collapse of such sources be of the Kerr type. The formalism dubbed horizon quantum mechanics (HQM) [1-8], was initially proposed with the purpose of describing the gravitational radius of spherically symmetric compact sources and determining the existence of a horizon in a quantum mechanical fashion. It therefore appears as a natural continuation in this research direction to extend the HQM to rotating sources. Unfortunately, this is not at all a conceptually trivial task.

In a classical spherically symmetric system, the gravitational radius is uniquely defined in terms of the (quasi-)local Misner-Sharp mass and it uniquely determines the location of the trapping surfaces where the null geodesic expansion vanishes. The latter surfaces are proper horizons in a timeindependent configuration, which is the case we shall always

\footnotetext{
a e-mail: casadio@bo.infn.it

be-mail: A.Giugno@physik.uni-muenchen.de

c e-mail: andrea.giusti@bo.infn.it

de-mail: octavian.micu@spacescience.ro
}

consider here. It is therefore rather straightforward to uplift this description of the causal structure of space-time to the quantum level by simply imposing the relation between the gravitational radius and the Misner-Sharp mass as an operatorial constraint to be satisfied by the physical states of the system [3].

In a non-spherical space-time, such as the one generated by an axially symmetric rotating source, although there are candidates for the quasi-local mass function that should replace the Misner-Sharp mass [9], the locations of trapping surfaces, and horizons, remain to be determined separately. We shall therefore consider a different path and simply uplift to a quantum condition the classical relation of the two horizon radii with the mass and angular momentum of the source obtained from the Kerr metric. This extended HQM is clearly more heuristic than the one employed for the spherically symmetric systems, but we note that it is indeed fully consistent with the expected asymptotic structure of axially symmetric space-times.

Beside the formal developments, we shall also apply the extended HQM to specific states with non-vanishing angular momentum of the harmonic black hole model introduced in Ref. [10]. ${ }^{1}$ This model can be considered as a working realization of the corpuscular black holes proposed by Dvali and Gomez [12-19], and it turns out to be simple enough, so as to allow one to determine explicitly the probability that the chosen states are indeed black holes. Furthermore, we will investigate the existence of the inner horizon and likelihood of extremal configurations for these states.

The paper is organized as follows: at the beginning of Sect. 2, we briefly summarize the HQM and recall some of the main results obtained for static spherically symmetric sources; the extension of the existing formalism to the case of stationary axisymmetric sources, which are both localized in space and subject to a motion of pure rotation, is presented

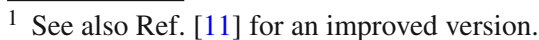


in Sect. 2.2; a short survey of the harmonic model for corpuscular black holes is given in Sect. 3, where we then discuss some elementary applications of the HQM to rotating black holes whose quantum state contains a large number of (toy) gravitons; finally, in Sect. 4, we conclude with remarks and hints for future research.

\section{Horizon quantum mechanics}

We start from reviewing the basics of the (global) HQM for static spherically symmetric sources [1-8], and then extend this formalism to rotating systems by means of the Kerr relation for the horizon radii in terms of the asymptotic mass and angular momentum of the space-time. In particular, we shall rely on the results for the "global" case of Ref. [3] and follow closely the notation therein.

\subsection{Spherically symmetric systems}

The general spherically symmetric metric $g_{\mu \nu}$ can be written $\mathrm{as}^{2}$

$\mathrm{d} s^{2}=g_{i j} \mathrm{~d} x^{i} \mathrm{~d} x^{j}+r^{2}\left(x^{i}\right)\left(\mathrm{d} \theta^{2}+\sin ^{2} \theta \mathrm{d} \phi^{2}\right)$,

where $r$ is the areal coordinate and $x^{i}=\left(x^{1}, x^{2}\right)$ are coordinates on surfaces of constant angles $\theta$ and $\phi$. The location of a trapping surface is then determined by the equation

$g^{i j} \nabla_{i} r \nabla_{j} r=0$,

where $\nabla_{i} r$ is perpendicular to surfaces of constant area $\mathcal{A}=$ $4 \pi r^{2}$. If we set $x^{1}=t$ and $x^{2}=r$, and if we denote the static matter density by $\rho=\rho(r)$, the Einstein field equations tell us that

$g^{r r}=1-\frac{2 \ell_{p}\left(m / m_{p}\right)}{r}$,

where the Misner-Sharp mass is given by

$m(r)=4 \pi \int_{0}^{r} \rho(\bar{r}) \bar{r}^{2} \mathrm{~d} \bar{r}$,

as if the space inside the sphere were flat. A trapping surface then exists if there are values of $r$ such that the gravitational radius $r_{\mathrm{H}}=2 \ell_{p} m / m_{p} \geq r$. If this relation holds in the vacuum outside the region where the source is located, $r_{\mathrm{H}}$ becomes the usual Schwarzschild radius associated with the total Arnowitt-Deser-Misner (ADM) [20] mass $M=m(\infty)$,

$R_{\mathrm{H}}=2 \ell_{p} \frac{M}{m_{p}}$

${ }^{2}$ We shall use units with $c=1$, and the Newton constant $G=\ell_{p} / m_{p}$, where $\ell_{p}$ and $m_{p}$ are the Planck length and mass, respectively, and $\hbar=\ell_{p} m_{p}$. and the above argument gives a mathematical foundation to Thorne's hoop conjecture [21].

This description clearly becomes questionable for sources of the Planck size or lighter, for which quantum effects may not be neglected. The Heisenberg principle introduces an uncertainty in the spatial localization of the order of the Compton-de Broglie length, $\lambda_{M} \simeq \ell_{p} m_{p} / M$, and we could argue that $R_{\mathrm{H}}$ only makes sense if $R_{\mathrm{H}} \gtrsim \lambda_{M}$, that is, $M \gtrsim m_{p}$. The HQM was precisely proposed in order to describe cases in which one expects quantum uncertainties are not negligible. For this purpose, we assume the existence of two observables, the quantum Hamiltonian corresponding to the total energy $M$ of the system, ${ }^{3}$

$\hat{H}=\sum_{\alpha} E_{\alpha}\left|E_{\alpha}\right\rangle\left\langle E_{\alpha}\right|$,

where the sum is over the Hamiltonian eigenmodes, and the gravitational radius with eigenstates

$\hat{R}_{\mathrm{H}}\left|R_{\mathrm{H} \beta}\right\rangle=R_{\mathrm{H} \beta}\left|R_{\mathrm{H} \beta}\right\rangle$.

General states for our system can correspondingly be described by linear combinations of the form

$$
|\Psi\rangle=\sum_{\alpha, \beta} C\left(E_{\alpha}, R_{\mathrm{H} \beta}\right)\left|E_{\alpha}\right\rangle\left|R_{\mathrm{H} \beta}\right\rangle,
$$

but only those for which the relation (2.5) between the Hamiltonian and gravitational radius holds are viewed as physical. In particular, we impose (2.5) after quantization, as the weak Gupta-Bleuler constraint

$$
\begin{aligned}
0 & =\left(\hat{H}-\frac{m_{p}}{2 \ell_{p}} \hat{R}_{\mathrm{H}}\right)|\Psi\rangle \\
& =\sum_{\alpha, \beta}\left(E_{\alpha}-\frac{m_{p}}{2 \ell_{p}} R_{\mathrm{H} \beta}\right) C\left(E_{\alpha}, R_{\mathrm{H} \beta}\right)\left|E_{\alpha}\right\rangle\left|R_{\mathrm{H} \beta}\right\rangle .
\end{aligned}
$$

The solution is clearly given by

$C\left(E_{\alpha}, R_{\mathrm{H} \beta}\right)=C\left(E_{\alpha}, 2 \ell_{p} E_{\alpha} / m_{p}\right) \delta_{\alpha \beta}$,

which means that Hamiltonian eigenmodes and gravitational radius eigenmodes can only appear suitably paired in a physical state. The interpretation of this result is simply that the gravitational radius is not an independent degree of freedom in our treatment, precisely because of the constraint $(2.5){ }^{4}$

\footnotetext{
${ }^{3}$ See also Ref. [22] for further clarifications why $H$ is to be taken as the (super-)Hamiltonian of the ADM formalism. We will return to this important point in Sect. 3.

${ }^{4}$ For a comparison with different approaches to horizon quantization, see Sect. 2.4 in Ref. [8].
} 
By tracing out the gravitational radius part, we recover the spectral decomposition of the source wave function,

$$
\begin{aligned}
\left|\psi_{\mathrm{S}}\right\rangle & =\sum_{\gamma}\left\langle R_{\mathrm{H} \gamma}\left|\sum_{\alpha, \beta}\right| R_{\mathrm{H} \beta}\right\rangle C\left(E_{\alpha}, 2 \ell_{p} E_{\alpha} / m_{p}\right) \delta_{\alpha \beta}\left|E_{\alpha}\right\rangle \\
& =\sum_{\alpha} C\left(E_{\alpha}, 2 \ell_{p} E_{\alpha} / m_{p}\right)\left|E_{\alpha}\right\rangle \\
& \equiv \sum_{\alpha} C_{\mathrm{S}}\left(E_{\alpha}\right)\left|E_{\alpha}\right\rangle
\end{aligned}
$$

in which we used the (generalized) orthonormality of the gravitational radius eigenmodes [3]. Note that Eq. (2.10) now ensures that the result of this operation of integrating out the gravitational radius is still a pure quantum state.

Conversely, by integrating out the energy eigenstates, we will obtain the horizon wave function (HWF) [1-3]

$$
\begin{aligned}
\left|\psi_{\mathrm{H}}\right\rangle & =\sum_{\gamma}\left\langle E_{\gamma}\left|\sum_{\alpha, \beta}\right| E_{\beta}\right\rangle C\left(E_{\alpha}, 2 \ell_{p} E_{\alpha} / m_{p}\right) \delta_{\alpha \beta}\left|R_{\mathrm{H} \beta}\right\rangle \\
& =\sum_{\alpha} C_{\mathrm{S}}\left(m_{p} R_{\mathrm{H} \alpha} / 2 \ell_{p}\right)\left|R_{\mathrm{H} \alpha}\right\rangle
\end{aligned}
$$

Or

$$
\psi_{\mathrm{H}}\left(R_{\mathrm{H}_{\alpha}}\right)=\left\langle R_{\mathrm{H}_{\alpha}} \mid \psi_{\mathrm{H}}\right\rangle=C_{\mathrm{S}}\left(m_{p} R_{\mathrm{H}_{\alpha}} / 2 \ell_{p}\right),
$$

where $m_{p} R_{\mathrm{H} \alpha} / 2 \ell_{p}=E\left(R_{\mathrm{H} \alpha}\right)$ is fixed by the constraint (2.5). If the index $\alpha$ is continuous (again, see Ref. [3] for some important remarks), the probability density that we detect a gravitational radius of size $R_{\mathrm{H}}$ associated with the quantum state $\left|\psi_{\mathrm{S}}\right\rangle$ is given by $\mathcal{P}_{\mathrm{H}}\left(R_{\mathrm{H}}\right)=$ $4 \pi R_{\mathrm{H}}^{2}\left|\psi_{\mathrm{H}}\left(R_{\mathrm{H}}\right)\right|^{2}$, and we can define the conditional probability density that the source lies inside its own gravitational radius $R_{\mathrm{H}}$ as

$\mathcal{P}_{<}\left(r<R_{\mathrm{H}}\right)=P_{\mathrm{S}}\left(r<R_{\mathrm{H}}\right) \mathcal{P}_{\mathrm{H}}\left(R_{\mathrm{H}}\right)$,

where $P_{\mathrm{S}}\left(r<R_{\mathrm{H}}\right)=4 \pi \int_{0}^{R_{\mathrm{H}}}\left|\psi_{\mathrm{S}}(r)\right|^{2} r^{2} \mathrm{~d} r{ }^{5}$ Finally, the probability that the system in the state $\left|\psi_{\mathrm{S}}\right\rangle$ is a black hole will be obtained by integrating (2.14) over all possible values of $R_{\mathrm{H}}$, namely

$P_{\mathrm{BH}}=\int_{0}^{\infty} \mathcal{P}_{<}\left(r<R_{\mathrm{H}}\right) \mathrm{d} R_{\mathrm{H}}$.

Note that now the gravitational radius is necessarily "fuzzy" and characterized by an uncertainty $\Delta R_{\mathrm{H}}=\sqrt{\left\langle R_{\mathrm{H}}^{2}\right\rangle-\left\langle R_{\mathrm{H}}\right\rangle^{2}}$.

This quantum description for the total ADM mass $M$ and global gravitational radius $R_{\mathrm{H}}$ will be next extended to rotating sources by appealing to the asymptotic charges of axially symmetric space-times. We would like to recall that in Ref. [3] a local construction was also introduced based on the quasi-local mass (2.4), which allows one to describe quantum mechanically any trapping surfaces. However, that local

\footnotetext{
5 One can also view $\mathcal{P}_{<}\left(r<R_{\mathrm{H}}\right)$ as the probability density that the sphere $r=R_{\mathrm{H}}$ is a horizon.
}

analysis cannot be extended to rotating sources without a better understanding of the relation between quasi-local charges and the corresponding casual structure [9].

\subsection{Rotating sources: Kerr horizons}

Our aim is now to extend the HQM to rotating sources, for which there is no general consensus about the proper quasilocal mass function to employ, and how to determine the causal structure from it. For this reason, we shall explicitly consider relations that hold in space-times of the Kerr family, generated by stationary axisymmetric sources which are both localized in space and subject to a motion of pure rotation in the chosen reference frame.

We assume the existence of a complete set of commuting operators $\left\{\widehat{H}, \widehat{J}^{2}, \widehat{J_{z}}\right\}$ acting on a Hilbert space $\mathcal{H}$ connected with the quantum nature of the source. We also consider only the discrete part of the energy spectrum [3], and denote with $\alpha=\{a, j, m\}$ the set of quantum numbers parametrizing the spectral decomposition of the source, that is,

$$
\left|\psi_{S}\right\rangle=\sum_{a, j, m} C_{S}\left(E_{a j}, \lambda_{j}, \xi_{m}\right)|a j m\rangle,
$$

where the sum formally represents the spectral decomposition in terms of the common eigenmodes of the operators $\left\{\hat{H}, \hat{J}^{2}, \hat{J}_{z}\right\}$. In particular, we have ${ }^{6}$

$$
\begin{aligned}
\hat{H} & =\sum_{a, j, m} E_{a j}|a j m\rangle\langle a j m|, \\
\hat{J}^{2} & \equiv \frac{m_{p}^{2}}{\ell_{p}^{2}} \hat{j}^{2}=m_{p}^{4} \sum_{a, j, m} j(j+1)|a j m\rangle\langle a j m| \\
& \equiv \sum_{a, j, m} \lambda_{j}|a j m\rangle\langle a j m|, \\
\hat{J}_{z} & \equiv \frac{m_{p}}{\ell_{p}} \hat{j}_{z}=m_{p}^{2} \sum_{a, j, m} m|a j m\rangle\langle a j m| \\
& \equiv \sum_{a, j, m} \xi_{m}|a j m\rangle\langle a j m| .
\end{aligned}
$$

From the previous discussion, one can also easily infer that $j \in \mathbb{N}_{0} / 2, m \in \mathbb{Z} / 2$, with $|m| \leq j$, and $a \in \mathcal{I}$, where $\mathcal{I}$ is a discrete set of labels that can be either finite of infinite.

Let us first note that Eq. (2.16) stems from the idea that the space-time should reflect the symmetries of the source. Therefore, our first assumption is that the source should obviously have an angular momentum in order to describe a rotating black hole. Now, for a stationary asymptotically flat space-time, we can still define the ADM mass $M$ and, following Ref. [3] as outlined in the previous subsection, we

\footnotetext{
${ }^{6}$ For later convenience, we rescale the standard angular momentum operators $\hat{j}^{2}$ and $\hat{j}_{z}$ by factors of $G_{N}$ so as to have all operators proportional to $m_{p}$ to a suitable power.
} 
can replace this classical quantity with the expectation value of our Hamiltonian, ${ }^{7}$

$$
\begin{aligned}
M & \rightarrow\left\langle\psi_{\mathrm{S}}|\hat{H}| \psi_{\mathrm{S}}\right\rangle \\
= & \sum_{a, j, m} \sum_{b, k, n} C_{S}^{*}\left(E_{a j}, \lambda_{j}, \xi_{m}\right) \\
& \times C_{S}\left(E_{b k}, \lambda_{k}, \xi_{n}\right)\langle a j m|\widehat{H}| b k n\rangle \\
= & \sum_{a, j, m}\left|C_{S}\left(E_{a j}, \lambda_{j}, \xi_{m}\right)\right|^{2} E_{a j} .
\end{aligned}
$$

In general relativity, we can also define a conserved classical charge arising from the axial symmetry by means of the Komar integral. This will be the total angular momentum $J$ of the Kerr space-time. However, in our description of the quantum source, we have two distinct notions of angular momentum, i.e. the total angular momentum

$$
\left\langle\psi_{\mathrm{S}}\left|\hat{J}^{2}\right| \psi_{\mathrm{S}}\right\rangle=\sum_{a, j, m}\left|C_{S}\left(E_{a j}, \lambda_{j}, \xi_{m}\right)\right|^{2} \lambda_{j},
$$

and the component of the angular momentum along the axis of symmetry

$\left\langle\psi_{\mathrm{S}}\left|\hat{J}_{z}\right| \psi_{\mathrm{S}}\right\rangle=\sum_{a, j, m}\left|C_{S}\left(E_{a j}, \lambda_{j}, \xi_{m}\right)\right|^{2} \xi_{m}$

Since, at least classically, we can always rotate our reference frame so that the axis of symmetry is along the $z$ axis, it is reasonable to consider $\hat{J}^{2}$ as the quantum extension of the classical angular momentum for a Kerr black hole,

$J^{2} \rightarrow\left\langle\psi_{\mathrm{S}}\left|\hat{J}^{2}\right| \psi_{\mathrm{S}}\right\rangle=\sum_{a, j, m}\left|C_{S}\left(E_{a j}, \lambda_{j}, \xi_{m}\right)\right|^{2} \lambda_{j}$.

In the following, we will further assume that $\left\langle\hat{J}_{z}\right\rangle$ is maximum in our quantum states, so that the proper (semi-)classical limit is recovered, that is,

$\left\langle\psi_{\mathrm{S}}\left|\left(\hat{J}^{2}-\hat{J}_{z}^{2}\right)\right| \psi_{\mathrm{S}}\right\rangle \underset{\hbar \rightarrow 0}{\stackrel{j \rightarrow \infty}{\rightarrow}} 0$,

for $\hbar j=\ell_{p} m_{p} j$ held constant.

For the Kerr space-time we have two horizons given by

$R_{\mathrm{H}}^{( \pm)}=\frac{\ell_{p}}{m_{p}}\left(M \pm \sqrt{M^{2}-\frac{J^{2}}{M^{2}}}\right)$,

provided $J^{2}<M^{4}$. Let us then introduce two operators $\hat{R}^{( \pm)}$ and, for the sake of brevity, write their eigenstates as

$\hat{R}_{\mathrm{H}}^{( \pm)}|\beta\rangle_{ \pm}=R_{\mathrm{H}}^{( \pm)}|\beta\rangle_{ \pm}$.

The generic state for our system can now be described by a triply entangled state given by

\footnotetext{
${ }^{7}$ See footnote 3 .
}

$$
\begin{aligned}
|\Psi\rangle= & \sum_{a, j, m} \sum_{\alpha, \beta} C\left(E_{a j}, \lambda_{j}, \xi_{m}, R_{\mathrm{H}_{\alpha}}^{(+)},\right. \\
& R_{\mathrm{H}_{\beta}^{(-)}}^{(-)}|a j m\rangle|\alpha\rangle_{+}|\beta\rangle_{-},
\end{aligned}
$$

but Eq. (2.25) tells us that in order to be able to define the analog of the condition (2.9) for the rotating case, we have to assume some mathematical restrictions on the operator counterparts of $M$ and $J$. First of all, the term $J^{2} / M^{2}$ tells us that we should assume $\hat{H}$ to be an invertible self-adjoint operator, so that

$J^{2} / M^{2} \rightarrow \hat{J}^{2}\left(\hat{H}^{-1}\right)^{2}=\left(\hat{H}^{-1}\right)^{2} \hat{J}^{2}$.

For this purpose, it is useful to recall a corollary of the spectral theorem:

Corollary 2.1 Let $\hat{A}$ be a self-adjoint positive semi-definite operator. Then $\hat{A}$ has a positive semi-definite square root $\hat{S}$, that is, $\hat{S}$ is self-adjoint, positive semi-definite, and

$\hat{S}^{2}=\hat{A}$.

If $\hat{A}$ is positive definite, then $\hat{S}$ is positive definite.

It follows that the operator $\hat{H}^{2}-\hat{J}^{2}\left(\hat{H}^{-1}\right)^{2}$ should be, at least, a positive semi-definite operator. On defining the operators

$\hat{\mathcal{O}}^{ \pm} \equiv \hat{H} \pm\left(\hat{H}^{2}-\hat{J}^{2} \hat{H}^{-2}\right)^{1 / 2}$,

we see that the physical states of the system are those simultaneously satisfying

$\left(\hat{R}_{\mathrm{H}}^{(+)}-\hat{\mathcal{O}}^{+}\right)|\Psi\rangle_{\text {phys }}=0$

and

$\left(\hat{R}_{\mathrm{H}}^{(-)}-\hat{\mathcal{O}}^{-}\right)|\Psi\rangle_{\text {phys }}=0$.

These two conditions reduce to

$$
\begin{aligned}
& C\left(\{a j m\}, R_{\mathrm{H}_{\alpha}}^{(+)}, R_{\mathrm{H}_{\beta}}^{(-)}\right) \\
& \quad=C\left(E_{a j},\{j m\}, R_{\mathrm{H}_{a j}}^{(+)}\left(E_{a j}\right), R_{\mathrm{H}_{\beta}}^{(-)}\right) \delta_{\alpha,\{a, j\}}, \\
& C\left(\{a j m\}, R_{\mathrm{H}_{\alpha}}^{(+)}, R_{\mathrm{H}_{\beta}}^{(-)}\right) \\
& \quad=C\left(E_{a j},\{j m\}, R_{\mathrm{H}_{\alpha}}^{(+)}, R_{\mathrm{H}_{a j}}^{(-)}\left(E_{a j}\right)\right) \delta_{\beta,\{a, j\}},
\end{aligned}
$$

from which we obtain

$$
\begin{aligned}
& C\left(E_{a j},\{j m\}, R_{\mathrm{H}_{\alpha}}^{(+)}, R_{\mathrm{H}_{\beta}}^{(-)}\right) \\
& =C\left(E_{a j},\{j m\},\right. \\
& \left.\quad R_{\mathrm{H}_{a j}}^{(+)}\left(E_{a j}\right), R_{\mathrm{H}_{a j}^{(-)}}^{(-)}\left(E_{a j}\right)\right) \delta_{\alpha,\{a, j\}} \delta_{\beta,\{a, j\}} .
\end{aligned}
$$

By tracing out the geometric parts, we should recover the matter state, that is,

$$
\begin{aligned}
\left|\psi_{\mathrm{S}}\right\rangle= & \sum_{a, j, m} C\left(E_{a j}, \lambda_{j}, \xi_{m}, R_{\mathrm{H}_{a j}}^{(+)}\left(E_{a j}\right),\right. \\
& \left.R_{\mathrm{H}_{a j}^{(-)}}^{(-)}\left(E_{a j}\right)\right)|a j m\rangle,
\end{aligned}
$$


which implies

$$
\begin{aligned}
& C_{S}\left(E_{a j}, \lambda_{j}, \xi_{m}\right) \\
& \quad=C\left(E_{a j}, \lambda_{j}, \xi_{m}, R_{\mathrm{H}_{a j}}^{(+)}\left(E_{a j}\right), R_{\mathrm{H}_{a j}}^{(-)}\left(E_{a j}\right)\right) .
\end{aligned}
$$

Now, by integrating out the matter state, together with one of the two geometric parts, we can compute the wave function corresponding to each horizon,

$\psi_{ \pm}\left(R_{\mathrm{H}}^{( \pm)}\right)=C\left(E_{a j}\left(R_{\mathrm{H}}^{( \pm)}\right), \lambda_{j}\left(R_{\mathrm{H}}^{( \pm)}\right), \xi_{m}\left(R_{\mathrm{H}}^{( \pm)}\right)\right)$.

It is also important to stress that the Hamiltonian constraints imply a strong relation between the two horizons, indeed we have $R_{H}^{ \pm}=R_{H}^{ \pm}\left(R_{H}^{\mp}\right)$.

\section{Corpuscular harmonic black holes}

In the corpuscular model proposed by Dvali and Gomez [1219], black holes are macroscopic quantum objects made of gravitons with a very large occupation number $N$ in the ground state, effectively forming Bose-Einstein condensates. As also derived in Ref. [22] from a post-Newtonian analysis of the coherent state of gravitons generated by a matter source, the virtual gravitons forming the black hole of radius $R_{\mathrm{H}}$ are "marginally bound" by their Newtonian potential energy $U$, that is,

$\mu+U_{N} \simeq 0$,

where $\mu$ is the graviton effective mass related to their quantum mechanical size via the Compton/de Broglie wavelength $\lambda_{\mu} \simeq \ell_{p} m_{p} / \mu$, and $\lambda_{\mu} \simeq R_{\mathrm{H}}$.

A first rough approximation for the potential energy $U_{N}$ is obtained by considering a square well for $r<\lambda_{\mu}$,

$U \simeq-N \alpha \frac{\hbar}{\lambda_{\mu}} \Theta(\ell-r)$,

where $\Theta$ is the Heaviside step function and the coupling constant $\alpha=\ell_{p}^{2} / \lambda_{\mu}^{2}=\mu^{2} / m_{p}^{2}$. The energy balance (3.1) then leads to $N \alpha=1$ and, with $\lambda_{\mu} \simeq R_{\mathrm{H}}$,

$\mu \simeq m_{p} \frac{\ell_{p}}{R_{\mathrm{H}}} \simeq \frac{m_{p}}{\sqrt{N}}$,

so that

$M \simeq N \mu \simeq \sqrt{N} m_{p}$

A better approximation for the potential energy was employed in Ref. [10], which takes the harmonic form

$$
\begin{aligned}
V & =\frac{1}{2} \mu \omega^{2}\left(r^{2}-d^{2}\right) \Theta(d-r) \\
& \equiv V_{0}(r) \Theta(d-r),
\end{aligned}
$$

where the parameters $d$ and $\omega$ will have to be so chosen as to ensure the highest energy mode available to gravitons is just marginally bound [see Eq. (3.1)]. If we neglect the finite size of the well, the Schrödinger equation in spherical coordinates,

$$
\begin{aligned}
& \frac{\hbar^{2}}{2 \mu r^{2}}\left[\frac{\partial}{\partial r}\left(r^{2} \frac{\partial}{\partial r}\right)+\frac{1}{\sin \theta} \frac{\partial}{\partial \theta}\left(\sin \theta \frac{\partial}{\partial \theta}\right)+\frac{1}{\sin ^{2} \theta} \frac{\partial^{2}}{\partial \phi^{2}}\right] \psi \\
& =\left(V_{0}-\mathcal{E}\right) \psi,
\end{aligned}
$$

yields the well-known eigenfunctions

$$
\begin{aligned}
& \psi_{n j m}\left(r, \theta, \phi ; \lambda_{\mu}\right) \\
& =\mathcal{N} r^{l} \mathrm{e}^{-\frac{r^{2}}{2 \lambda_{\mu}^{2}}}{ }_{1} F_{1}\left(-n, l+3 / 2, r^{2} / \lambda_{\mu}^{2}\right) Y_{l m}(\theta, \phi),
\end{aligned}
$$

where $\mathcal{N}$ is a normalization constant, ${ }_{1} F_{1}$ the Kummer confluent hypergeometric function of the first kind and $Y_{l m}(\theta, \phi)$ are the usual spherical harmonics. The corresponding eigenvalues are given by

$\mathcal{E}_{n l}=\hbar \omega\left(2 n+l+\frac{3}{2}\right)+V_{0}(0)$,

where $n$ is the radial quantum number. It is important to remark that the quantum numbers $l$ and $m$ here must not be confused with the total angular momentum numbers $j$ and $m$ of Sect. 2.2, as the latter are the sum of the former. At the same time, the "energy" eigenvalues $\mathcal{E}_{n l}$ must not be confused with the ADM energy $E_{a j}$ of that section, here equal to $N \mu$ by construction.

If we denote with $n_{0}$ and $l_{0}$ the quantum numbers of the highest "energy" state, and include the graviton effective mass $\mu$ in the constant $V_{0}(0)$, the condition (3.1) becomes $\mathcal{E}_{n_{0} l_{0}} \simeq 0$, or

$V_{0}(0) \simeq-\hbar \omega\left(2 n_{0}+l_{0}+\frac{3}{2}\right)$,

which yields

$\omega d^{2} \simeq 2 \frac{\hbar}{\mu}\left(2 n_{0}+l_{0}+\frac{3}{2}\right)$.

We now further assume that $d \simeq \lambda_{\mu} \simeq R_{\mathrm{H}}^{(+)}$and use the Compton relation for $\mu$, so that the above relation fully determines

$\omega \simeq \frac{2}{\lambda_{\mu}}\left(2 n_{0}+l_{0}+\frac{3}{2}\right)$.

The potential can be finally written as

$V_{0}=2 \mu\left(2 n_{0}+l_{0}+\frac{3}{2}\right)^{2} \frac{r^{2}-\lambda_{\mu}^{2}}{\lambda_{\mu}^{2}}$

and the eigenvalues as

$$
\begin{aligned}
\mathcal{E}_{n l} & \simeq-\hbar \omega\left[2\left(n_{0}-n\right)+\left(l_{0}-l\right)\right] \\
& \simeq-2 \mu\left(2 n_{0}+l_{0}+\frac{3}{2}\right)\left[2\left(n_{0}-n\right)+\left(l_{0}-l\right)\right] \\
& \equiv-2 \mu_{0}\left[2\left(n_{0}-n\right)+\left(l_{0}-l\right)\right]
\end{aligned}
$$


which of course holds only for $n \leq n_{0}$ and $l \leq l_{0}$. Let us remark that the fact the above "energy" is negative for the allowed values of $n$ and $j$ is indeed in agreement with the post-Newtonian analysis of the "maximal packing condition" for the virtual gravitons in the black hole [22]. ${ }^{8}$

In the following, we shall consider a few specific states in order to show the kind of results one can obtain from the general HQM formalism of Sect. 2.2 applied to harmonic models of spinning black holes.

\subsection{Rotating black holes}

We shall now consider some specific configurations of harmonic black holes with angular momentum and apply the extended HQM described in the previous section. We first remark that the quantum state of $N$ identical gravitons will be a $N$-particle state, i.e. a vector of the $N$-particle Fock space $\mathcal{F}=\mathcal{H}^{\otimes N}$, where $\mathcal{H}$ is a suitable 1-particle Hilbert space. However, both the Hamiltonian of the system $\hat{H}$ and the gravitational radius $\hat{R}_{\mathrm{H}}$ are global observables and act as $N$-body operators on $\mathcal{F}$.

\subsubsection{Single eigenstates}

The simplest configuration corresponds to all toy gravitons in the same mode, and the quantum state of the system is therefore given by

$$
|\Psi\rangle \equiv|M J\rangle=\bigotimes_{\alpha=1}^{N}|g\rangle_{\alpha},
$$

where $|g\rangle$ represents the wave function of a single component. In particular, this $|\Psi\rangle$ is a Hamiltonian eigenstate, for which the total ADM energy is simply given by

$$
\langle\Psi|\hat{H}| \Psi\rangle \equiv\langle\hat{H}\rangle=N \mu=M,
$$

and each graviton is taken in one of the modes (3.7). For the sake of simplicity, we shall set $n=n_{0}=0, l=l_{0}=2$ and $m= \pm 2$, that is

$$
\begin{aligned}
\langle r, \theta, \phi \mid g\rangle & =\psi_{02 \pm 2}\left(r, \theta, \phi ; \lambda_{\mu}\right) \\
& =\mathcal{N} r^{2} \exp \left(-\frac{r^{2}}{2 \lambda_{\mu}^{2}}\right) Y_{2 \pm 2}(\theta, \phi),
\end{aligned}
$$

where the normalization constant $\mathcal{N}=4 /\left(\sqrt{15} \pi^{1 / 4} \lambda_{\mu}^{7 / 2}\right)$. The total angular momentum is thus given by

$$
\begin{aligned}
\left\langle\Psi\left|\hat{J}^{2}\right| \Psi\right\rangle \equiv\left\langle\hat{J}^{2}\right\rangle & =4\left(N_{+}-N_{-}\right)\left(N_{+}-N_{-}+1 / 2\right) m_{p}^{2} \\
& \equiv 4 L^{2} N^{2} m_{p}^{2},
\end{aligned}
$$

\footnotetext{
$\overline{8}$ It becomes positive if we consider the effective mass $\mu<0$ for virtual gravitons.
}

where $N_{+} \geq N_{-}=N-N_{+}$is the number of spin up constituents (with $m=+2$ ). We also introduced the constant

$$
\begin{aligned}
L^{2} & =\left(\frac{N_{+}}{N}-\frac{N_{-}}{N}\right)\left(\frac{N_{+}}{N}-\frac{N_{-}}{N}+\frac{1}{2 N}\right) \\
& \equiv\left(2 n_{+}-1\right)\left(2 n_{+}-1+\frac{1}{2 N}\right) \simeq\left(2 n_{+}-1\right)^{2},
\end{aligned}
$$

where the approximate expression holds for $N \gg 1$. Note that $L^{2}=0$ for $n_{+} \equiv N_{+} / N=1 / 2$ (the non-rotating case with $\left.N_{+}=N_{-}\right)$and grows to a maximum $L^{2} \simeq 1+\mathcal{O}(1 / N)$ for the maximally rotating case $n_{+}=1$ ( or $N_{+}=N$ ).

Since we are considering an eigenstate of both the Hamiltonian $\hat{H}$ and the total angular momentum $\hat{J}^{2}$, the wave functions (2.37) for the two horizons will reduce to single eigenstates of the respective gravitational radii as well. In particular, replacing the above values into (2.30) and (2.31) yields

$\left\langle\hat{R}_{\mathrm{H}}^{( \pm)}\right\rangle=N \ell_{p} \frac{\mu}{m_{p}}\left(1 \pm \sqrt{1-4 \frac{L^{2} m_{p}^{4}}{N^{2} \mu^{4}}}\right)$.

The classical condition for the existence of these horizons is that the square root be real, which implies

$\mu^{2} \geq 2 m_{p}^{2} \frac{L}{N}$

The above bound vanishes for $N_{+}=N_{-}=N / 2$, as expected for a spherical black hole, and is maximum for $N_{+}=N$, in which case it yields

$\mu^{2} \gtrsim 2 \frac{m_{p}^{2}}{N}$

again for $N \gg 1$.

Since we are modeling black holes, it is particularly interesting to study in detail the consequences of assuming that all the constituents of our system lie inside the outer horizon. In other words, we next require that the Compton length of gravitons, $\lambda_{\mu}=\ell_{p} m_{p} / \mu$, is such that the modes (3.16) are mostly found inside the outer horizon radius $\left\langle\hat{R}_{\mathrm{H}}^{(+)}\right\rangle$. In order to impose this condition, we compute the singleparticle probability density

$$
\begin{aligned}
\mathcal{P}_{02}\left(r ; \lambda_{\mu}\right) & =\int_{-1}^{+1} \mathrm{~d} \cos \theta \int_{0}^{2 \pi} \mathrm{d} \phi\left|\psi_{02+2}\left(r, \theta, \phi ; \lambda_{\mu}\right)\right|^{2} \\
& =\mathcal{N}^{2} r^{6} \exp \left(-\frac{r^{2}}{\lambda_{\mu}^{2}}\right)
\end{aligned}
$$

where we used $\left|\psi_{02+2}\right|^{2}=\left|\psi_{02-2}\right|^{2}$. From Fig. 1, we then see that this probability is peaked well inside $\left\langle\hat{R}_{\mathrm{H}}^{(+)}\right\rangle$for $\lambda_{\mu}=$ $\left\langle\hat{R}_{\mathrm{H}}^{(+)}\right\rangle / 4$, whereas $\lambda_{\mu}=\left\langle\hat{R}_{\mathrm{H}}^{(+)}\right\rangle / 2$ is already borderline and $\lambda_{\mu}=\left\langle\hat{R}_{\mathrm{H}}^{(+)}\right\rangle$is clearly unacceptable. 


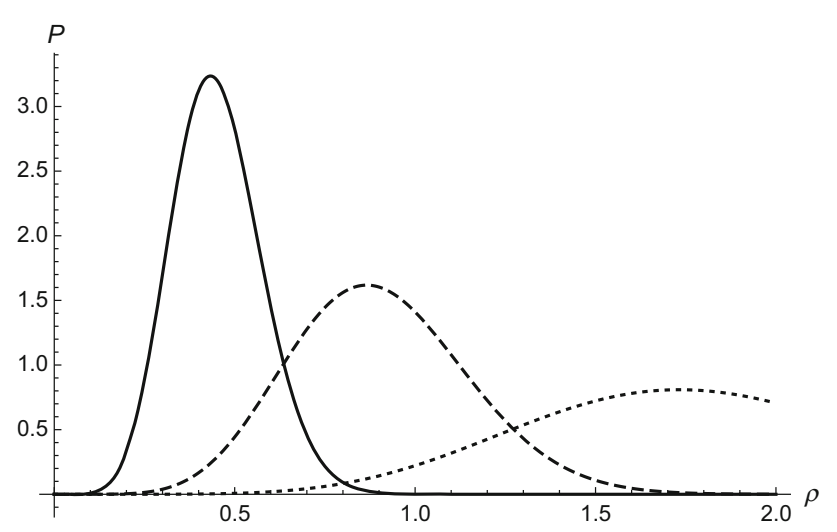

Fig. 1 Plots of $\mathcal{P}_{02}\left(\rho ; \lambda_{\mu}\right)$ as a function of $\rho=r /\left\langle\hat{R}_{\mathrm{H}}^{(+)}\right\rangle$for $\lambda_{\mu}=$ $\left\langle\hat{R}_{\mathrm{H}}^{(+)}\right\rangle / 4$ (solid line), $\lambda_{\mu}=\left\langle\hat{R}_{\mathrm{H}}^{(+)}\right\rangle / 2$ (dashed line) and $\lambda_{\mu}=\left\langle\hat{R}_{\mathrm{H}}^{(+)}\right\rangle$ (dotted line)

We find it in general convenient to introduce the variable

$\gamma\left(n_{+}, N\right) \equiv \frac{\left\langle\hat{R}_{\mathrm{H}}^{(+)}\right\rangle}{2 \lambda_{\mu}}$,

which should be at least 1 according to the above estimate, so that Eq. (3.19) reads

$\frac{2 \gamma m_{p}^{2}}{N \mu^{2}} \simeq 1+\sqrt{1-\frac{4 L^{2} m_{p}^{4}}{N^{2} \mu^{4}}}$

which we can solve for $x=2 \gamma m_{p}^{2} /\left(N \mu^{2}\right)$, that is,

$(x-1)^{2} \simeq 1-\ell^{2} x^{2}$

with the condition $\ell x \equiv(L / \gamma) x \leq 1$ to ensure the existence of the square root. The only positive solution is given by

$x \simeq \frac{2}{1+\ell^{2}}$

for which the existence condition reads $(\ell-1)^{2} \geq 0$ and is identically satisfied. The effective mass is then given by

$\mu^{2}=\frac{2 \gamma m_{p}^{2}}{N x} \simeq \frac{\gamma^{2}+L^{2}}{\gamma N} m_{p}^{2}$.

As a function of $N / 2 \leq N_{+} \leq N$, the above squared mass interpolates almost linearly between $\mu_{0}^{2}=\gamma m_{p}^{2} / N$ for $N_{+}=N_{-}=N / 2$ (so that $\left.L^{2}=0\right)$ and $\bar{\mu}^{2} \simeq(1+$ $\left.\gamma^{2}\right) m_{p}^{2} /(\gamma N)$ for the maximally rotating case $N_{+}=N \gg 1$ (for which $L^{2} \simeq 1$ ). The Compton length reads

$\lambda_{\mu}=\ell_{p} \frac{m_{p}}{\mu} \simeq \sqrt{\frac{\gamma N}{\gamma^{2}+L^{2}}} \ell_{p}$

the ADM mass is

$M=N \mu \simeq \sqrt{\frac{\gamma^{2}+L^{2}}{\gamma} N} m_{p}$. and the angular momentum

$$
\left\langle\hat{J}^{2}\right\rangle \simeq 4 N^{2} L^{2} m_{p}^{4} \simeq \frac{4 \gamma^{2} L^{2} M^{4}}{\left(\gamma^{2}+L^{2}\right)^{2}}<M^{4},
$$

for all values of $L \geq 0$. This seems to suggest that $N$ constituents of effective mass $\mu \sim m_{p} / \sqrt{N}$ cannot exceed the classical bound for black holes, or that naked singularities cannot be associated with such multi-particle states. However, a naked singularity has no horizon and we lose the condition (3.1) from which the effective mass $\mu$ is determined. If naked singularities can still be realized in the quantum realm, they must be described in a qualitatively different way from the present one. ${ }^{9}$

Let us now plug the effective mass (3.27) into Eq. (3.19),

$$
\begin{aligned}
\left\langle\hat{R}_{\mathrm{H}}^{( \pm)}\right\rangle & \simeq \ell_{p} \sqrt{\frac{\gamma^{2}+L^{2}}{\gamma} N}\left(1 \pm \sqrt{1-\frac{4 \gamma^{2} L^{2}}{\left(\gamma^{2}+L^{2}\right)^{2}}}\right) \\
& \simeq \ell_{p} \sqrt{\frac{N / \gamma}{\gamma^{2}+L^{2}}}\left(\gamma^{2}+L^{2} \pm\left|\gamma^{2}-L^{2}\right|\right) .
\end{aligned}
$$

One has $L^{2}=\gamma^{2}$ for

$n_{+}=n_{\mathrm{c}} \equiv \frac{2 N-1+\sqrt{1+4 \gamma^{2} N^{2}}}{4 N} \simeq \frac{1+\gamma}{2}-\frac{1}{4 N}$.

Since $1 / 2 \leq n_{+} \leq 1$, the critical value $n_{\mathrm{c}}$ becomes relevant only for $\gamma \simeq N \simeq 1$. For $N \gg 1$ and $\gamma \gtrsim 1$, the horizon radii are thus given by

$\left\langle\hat{R}_{\mathrm{H}}^{(-)}\right\rangle \simeq \frac{L^{2}}{\gamma^{2}}\left\langle\hat{R}_{\mathrm{H}}^{(+)}\right\rangle \simeq 2 L^{2} \ell_{p} \sqrt{\frac{N / \gamma}{\gamma^{2}+L^{2}}}$,

and $2 \gamma \lambda_{\mu} \simeq\left\langle\hat{R}_{\mathrm{H}}^{(+)}\right\rangle$, as we required. The above horizon structure for $1 / 2 \leq n_{+} \leq 1$ is displayed for $\gamma=2$ and $N=100$ in Fig. 2, where we also recall that $\lambda_{\mu}=\left\langle\hat{R}_{\mathrm{H}}^{(+)}\right\rangle / 4$.

It is particularly interesting to note that the extremal Kerr geometry can only be realized in our model if $\gamma$ is sufficiently small. In fact, $\left\langle\hat{R}_{\mathrm{H}}^{(-)}\right\rangle \simeq\left\langle\hat{R}_{\mathrm{H}}^{(+)}\right\rangle$requires

$\gamma^{2} \simeq L^{2}$

For $\gamma=1$ and $N=100$, the horizon structure is displayed in Fig. 3, where we see that the two horizons meet at $L^{2} \simeq 1$, that is, the configuration with $n_{+} \simeq 1$ in which (almost) all constituents are aligned. Note also that, technically, for $N \simeq 1$ and $\gamma$ small, there would be a finite range $n_{\mathrm{c}}<$ $n_{+} \leq 1$ in which the expressions of the two horizon radii switch. However, this result is clearly more dubious as one would be dealing with a truly quantum black hole made of a few constituents just loosely confined. Such configurations could play a role in the formation of black holes, or in the

${ }^{9}$ See Refs. [6,7] for spherically symmetric charged sources. 


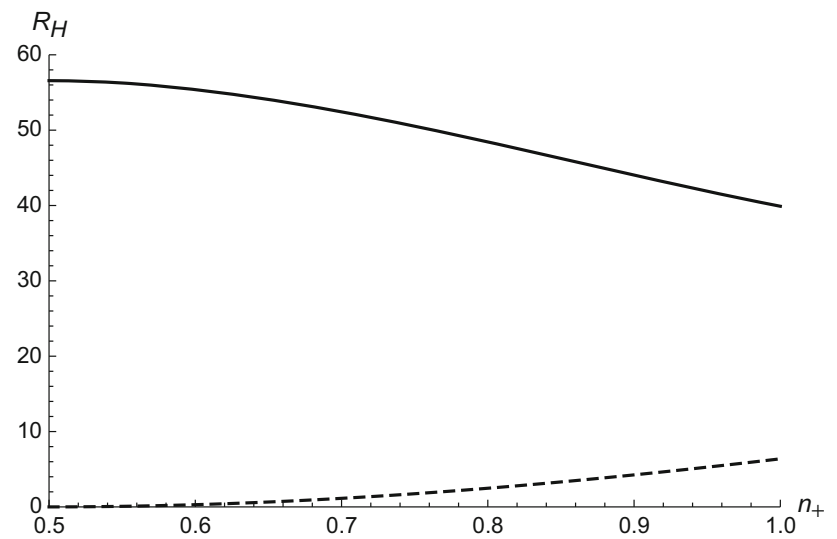

Fig. 2 Horizon radius $\left\langle\hat{R}_{\mathrm{H}}^{(+)}\right\rangle$(solid line) and $\left\langle\hat{R}_{\mathrm{H}}^{(-)}\right\rangle$(dashed line) in Planck length units for $N=100$ and $\gamma=2$

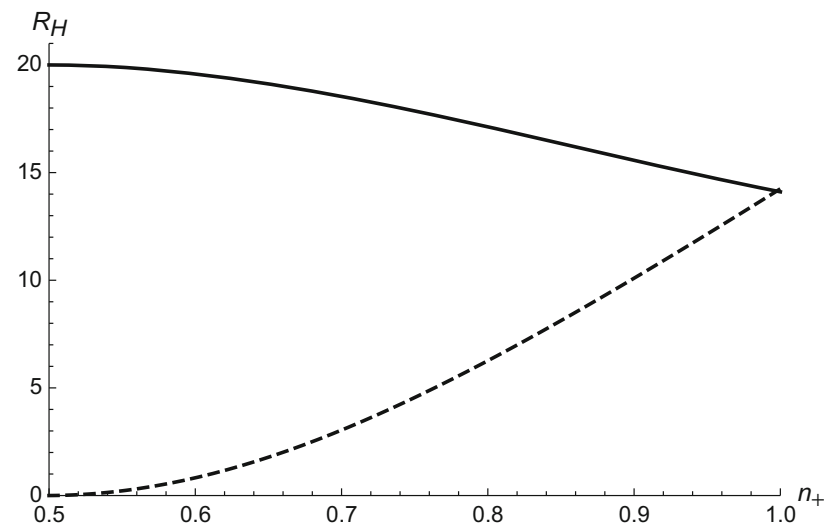

Fig. 3 Horizon radius $\left\langle\hat{R}_{\mathrm{H}}^{(+)}\right\rangle$(solid line) and $\left\langle\hat{R}_{\mathrm{H}}^{(-)}\right\rangle$(dashed line) in Planck length units for $N=100$ and $\gamma=1$

final stages of their evaporation, but we shall not consider this possibility any further here.

Finally, let us apply the HQM and compute the probability (2.15) that the system discussed above is indeed a black hole. We first note that, since we are considering eigenstates of the gravitational radii, the wave function (2.37) for the outer horizon will just contribute a Dirac delta peaked on the outer expectation value (3.19) to the general expression (2.14), that is,

$\mathcal{P}_{(+)}\left(R_{\mathrm{H}}\right)=\delta\left(R_{\mathrm{H}}-\left\langle\hat{R}_{\mathrm{H}}^{(+)}\right\rangle\right)$.

This implies

$P_{\mathrm{BH}}\left(n_{+}, N\right)=P_{<}^{(+)}\left(r_{1}<\left\langle\hat{R}_{\mathrm{H}}^{(+)}\right\rangle, \ldots, r_{N}<\left\langle\hat{R}_{\mathrm{H}}^{(+)}\right\rangle\right)$.

Moreover, since

$$
\left\langle\mathbf{r}_{1}, \ldots, \mathbf{r}_{N} \mid \Psi\right\rangle=\prod_{\alpha=1}^{N}\left\langle\mathbf{r}_{\alpha} \mid g\right\rangle_{\alpha}=\prod_{\alpha=1}^{N} \psi_{02 \pm 2}\left(r_{\alpha}, \theta_{\alpha}, \phi_{\alpha} ; \lambda_{\mu}\right),
$$

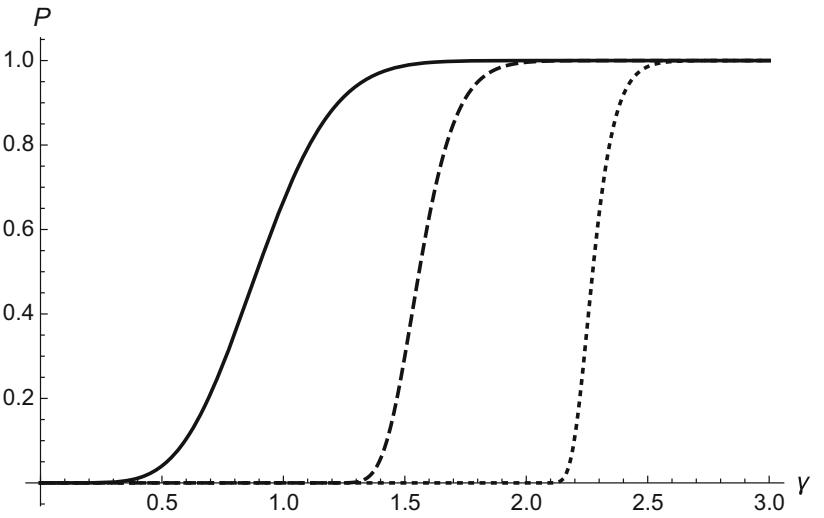

Fig. 4 Black hole probability (3.39) as a function of $\gamma=\left\langle\hat{R}_{\mathrm{H}}^{(+)}\right\rangle / 2 \lambda_{\mu}$ for $N=1$ (solid line), $N=10^{2}$ (dashed line) and $N=10^{6}$ (dotted line)

where $\mathbf{r} \equiv(r, \theta, \phi)$, the joint probability density in position space is simply given by

$$
\begin{aligned}
\mathcal{P}\left(r_{1}, \ldots, r_{N} ; \lambda_{\mu}\right) & =\prod_{\alpha=1}^{N} \mathcal{P}_{02}\left(r_{\alpha} ; \lambda_{\mu}\right) \\
& =\mathcal{N}^{2 N} r_{1}^{6} \cdots r_{N}^{6} \exp \left(-\frac{r_{1}^{2}+\cdots+r_{N}^{2}}{\lambda_{\mu}^{2}}\right),
\end{aligned}
$$

where we used Eq. (3.22). It immediately follows that

$P_{\mathrm{BH}}\left(n_{+}, N\right)=\prod_{\alpha=1}^{N} P_{<}^{(+)}\left(r_{\alpha}<\left\langle\hat{R}_{\mathrm{H}}^{(+)}\right\rangle\right)=\left[P_{<}^{(+)}\left(r<\left\langle\hat{R}_{\mathrm{H}}^{(+)}\right\rangle\right)\right]^{N}$,

with

$$
\begin{aligned}
P_{<}^{(+)}\left(r<\left\langle\hat{R}_{\mathrm{H}}^{(+)}\right\rangle\right)= & \int_{0}^{\left\langle\hat{R}_{\mathrm{H}}^{(+)}\right\rangle} \mathcal{P}_{02}\left(r ; \lambda_{\mu}\right) \mathrm{d} r \\
= & \operatorname{erf}(2 \gamma)-\frac{4}{15 \sqrt{\pi}} \gamma \\
& \times\left(64 \gamma^{4}+40 \gamma^{2}+15\right) \mathrm{e}^{-4 \gamma^{2}} \\
\equiv & P_{(+)}(\gamma)
\end{aligned}
$$

where we recall $\gamma$ was defined in Eq. (3.23), and depends on $N$ and $n_{+}$.

The single-particle $(N=1)$ black hole probability $P_{(+)}(\gamma)$ is represented by the solid line in Fig. 4, from which it is clear that it practically saturates to 1 for $\gamma \gtrsim 2$. The same graph shows that the minimum value of $\gamma$ for which $P_{\mathrm{BH}}\left(n_{+}, N\right)=\left[P_{(+)}(\gamma)\right]^{N}$ approaches 1 increases with $N$ (albeit very slowly). For instance, if we define $\gamma_{\mathrm{c}}$ as the value at which $P_{\mathrm{BH}}\left(n_{+}, N\right) \simeq 0.99$, we obtain the values of $\gamma_{\mathrm{c}}$ plotted in Fig. 5. It is also interesting to note that, for $\gamma=1$, which we saw can realize the extremal Kerr geometry, we 


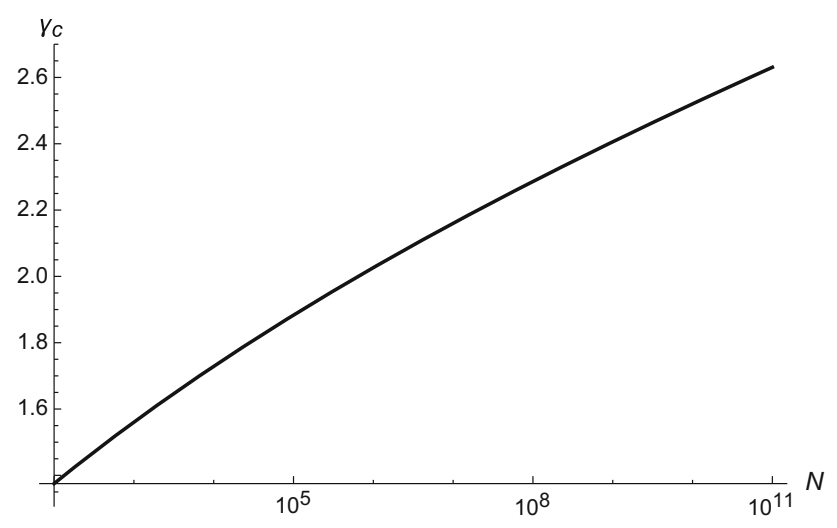

Fig. 5 Value of $\gamma_{\mathrm{c}}$ such that the black hole probability (3.39) is given by $P_{\mathrm{BH}}\left(n_{+}, N\right)=\left[P_{\mathrm{BH}}\left(\gamma \geq \gamma_{\mathrm{c}}\right)\right]^{N} \geq 99 \%$ for $N=10^{2}$ to $N=10^{11}$

find

$P_{\mathrm{BH}}\left(n_{+}, N\right) \simeq P_{\mathrm{BH}}(N) \simeq(0.67)^{N}$,

and the system is most likely not a black hole for $N \gg 1$, in agreement with the probability density shown in Fig. 1. One might indeed argue this probability is always too small for a (semi)classical black hole, and that the extremal Kerr configuration is therefore more difficult to achieve.

Analogously, we can compute the probability $P_{\mathrm{IH}}$ that the inner horizon is realized. Instead of Eq. (3.35), we now have

$\mathcal{P}_{(-)}\left(R_{\mathrm{H}}\right)=\delta\left(R_{\mathrm{H}}-\left\langle\hat{R}_{\mathrm{H}}^{(-)}\right\rangle\right)$,

which analogously leads to

$P_{\mathrm{IH}}\left(n_{+}, N\right)=\left[P_{<}^{(-)}\left(r<\left\langle\hat{R}_{\mathrm{H}}^{(-)}\right\rangle\right)\right]^{N}$.

It is then fairly obvious that, for any fixed value of $\gamma$, $P_{\mathrm{IH}}\left(n_{+}, N\right) \leq P_{\mathrm{BH}}\left(n_{+}, N\right)$ and that equality is reached at the extremal geometry with $\left\langle\hat{R}_{\mathrm{H}}^{(-)}\right\rangle \simeq\left\langle\hat{R}_{\mathrm{H}}^{(+)}\right\rangle$. Moreover, from $0 \leq L^{2} \leq 1$ and Eq. (3.33), we find $\left\langle\hat{R}_{\mathrm{H}}^{(-)}\right\rangle \lesssim$ $\left\langle\hat{R}_{\mathrm{H}}^{(+)}\right\rangle / \gamma^{2}$, so that for $\gamma=2$, the probability $P_{\mathrm{IH}} \lesssim(0.04)^{N}$ is totally negligible for $N \gg 1$. This suggest that the inner horizon can remain extremely unlikely even in configurations that should represent large (semi-)classical black holes.

\subsubsection{Superpositions}

The next step is investigating general superpositions of the states considered above,

$$
|\Psi\rangle=\sum_{i} a_{i}\left|M_{i} J_{i}\right\rangle
$$

where $\sum_{i}\left|a_{i}\right|^{2}=1$ and

$$
\left|M_{i} J_{i}\right\rangle=\bigotimes_{\alpha=1}^{N_{i}}\left|g_{i}\right\rangle_{\alpha}
$$

so that $M_{i}=N_{i} \mu_{i}$ and $J_{i}=\left(2 N_{i+}-N_{i}\right) j_{i} \equiv N_{i}\left(2 n_{i+}-\right.$ 1) $j_{i}$. One can repeat the same analysis as the one performed for the single-mode case, except that the two HWFs will now be superpositions of ADM values as well.

In practice, this means that Eqs. (3.35) and (3.42) are now replaced by

$\mathcal{P}_{( \pm)}\left(R_{\mathrm{H}}\right)=\sum_{i}\left|a_{i}\right|^{2} \delta\left(R_{\mathrm{H}}-R_{\mathrm{H}_{i}}^{( \pm)}\right)$,

where, from Eqs. (2.30) and (2.31), the horizon radii are given by

$R_{\mathrm{H}_{i}}^{( \pm)}=\ell_{p} \frac{M_{i}}{m_{p}}\left(1 \pm \sqrt{1-\frac{m_{p}^{4} J_{i}\left(J_{i}+1\right)}{M_{i}^{4}}}\right)$,

and the expectation values of the horizon radii are correspondingly given by

$\left\langle\hat{R}_{\mathrm{H}}^{( \pm)}\right\rangle=\sum_{i}\left|a_{i}\right|^{2} R_{\mathrm{H}_{i}}^{( \pm)}$.

As usual, we obtain the probability that the system is a black hole by considering the outer horizon, for which

$$
\begin{aligned}
P_{\mathrm{BH}} & =\sum_{i}\left|a_{i}\right|^{2} P_{<}^{(+)}\left(r_{1}<R_{\mathrm{H}_{i}}^{(+)}, \ldots, r_{N_{i}}<R_{\mathrm{H}_{i}}^{(+)}\right) \\
& =\sum_{i}\left|a_{i}\right|^{2}\left[P_{<}^{(+)}\left(r<R_{\mathrm{H}_{i}}^{(+)}\right)\right]^{N_{i}}
\end{aligned}
$$

where

$P_{<}^{(+)}\left(r<R_{\mathrm{H}_{i}}^{(+)}\right)=\int_{0}^{R_{\mathrm{H}_{i}}^{(+)}} \mathcal{P}_{n_{i} l_{i}}\left(r ; \lambda_{\mu_{i}}\right) \mathrm{d} r$,

and

$\mathcal{P}_{n l}\left(r ; \lambda_{\mu}\right)=\int_{-1}^{+1} \mathrm{~d} \cos \theta \int_{0}^{2 \pi} \mathrm{d} \phi\left|\psi_{n l \pm 2}\left(r, \theta, \phi ; \lambda_{\mu}\right)\right|^{2}$.

The explicit calculation of the above probability immediately becomes very cumbersome. For the purpose of exemplifying the kind of results one should expect, let us just consider a state

$$
|\Psi\rangle=\frac{a\left|M_{1} J_{1}\right\rangle+b\left|M_{2} J_{2}\right\rangle}{\sqrt{|a|^{2}+|b|^{2}}},
$$

where the two modes in superposition are given by $N$ constituents with quantum numbers $n_{1}=0, l_{1}=2$ and $m= \pm 2$ in the state (3.16), here denoted with $\left|g_{1}\right\rangle$; the same number $N$ of gravitons with quantum numbers $n_{2}=1, l_{2}=2$ and $m= \pm 2$ in the state

$$
\begin{aligned}
\left\langle\mathbf{r} \mid g_{2}\right\rangle & =\psi_{12 \pm 2}(r, \theta, \phi) \\
& =\mathcal{N}_{2} r^{2} \mathrm{e}^{-\frac{r^{2}}{2 \lambda_{\mu}^{2}}}\left(1-\frac{2 r^{2}}{7 \lambda_{\mu}^{2}}\right) Y_{2 \pm 2}(\theta, \phi),
\end{aligned}
$$




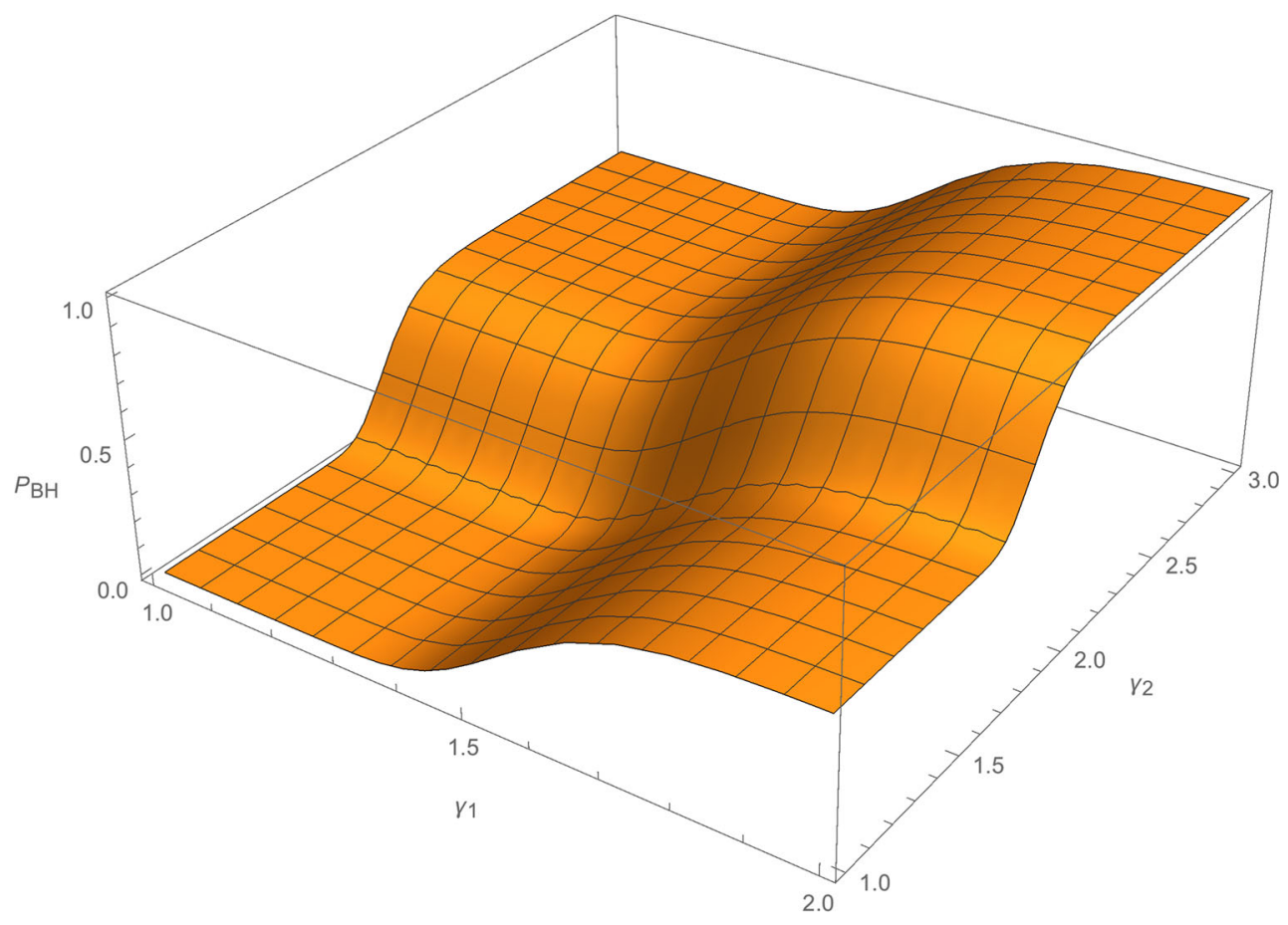

Fig. 6 Probability $P_{\mathrm{BH}}$ as a function of $\gamma_{1}=R_{\mathrm{H}_{1}}^{(+)} / 2 \lambda_{\mu}$ and $\gamma_{2}=R_{\mathrm{H}_{2}}^{(+)} / 2 \lambda_{\mu}$, for $a=b=1$ and $N=100$

where we further assumed that all constituents have the same Compton/de Broglie wavelength $\lambda_{\mu}$. It then follows that $M_{1}=M_{2} \equiv M$, so that

$R_{\mathrm{H}_{i}}^{( \pm)}=\ell_{p} \frac{M}{m_{p}}\left(1 \pm \sqrt{1-\frac{m_{p}^{4} J_{i}\left(J_{i}+1\right)}{M^{4}}}\right)$

and

$$
\begin{aligned}
\left\langle\hat{R}_{\mathrm{H}}^{( \pm)}\right\rangle= & \ell_{p} \frac{M}{m_{p}}\left[1 \pm \frac{1}{|a|^{2}+|b|^{2}}\left(|a|^{2} \sqrt{1-\frac{m_{p}^{4} J_{1}\left(J_{1}+1\right)}{M^{4}}}\right.\right. \\
& \left.+|b|^{2} \sqrt{\left.1-\frac{m_{p}^{4} J_{2}\left(J_{2}+1\right)}{M^{4}}\right)}\right],
\end{aligned}
$$

with each of the $J_{i}$ depending both on the numbers of spin up and the total number of constituents of each type, as defined in the beginning of this section. We also notice that when both $J_{1}$ and $J_{2}$ go to zero the expression simplifies to

$\left\langle\hat{R}_{\mathrm{H}}^{(+)}\right\rangle=\ell_{p} \frac{2 M}{m_{p}}$,

while $\left\langle\hat{R}_{\mathrm{H}}^{(-)}\right\rangle=0$, as expected for a Schwarzschild black hole.

The probability (3.49) can be computed explicitly and is shown in Fig. 6 for $N=100$, with $a=b=1$. Beside the specific shape of those curves, the overall result appears in line with what we found in the previous subsection for an Hamiltonian eigenstate: the system is most certainly a black hole provided the Compton/de Broglie length is sufficiently shorter than the possible outer horizon radius (that is, for sufficiently large $\gamma_{1}$ and $\gamma_{2}$ ).

\section{Conclusions}

After a brief review of the original HQM for static spherically symmetric sources, we have generalized this formalism in order to provide a proper framework for the study of quantum properties of the causal structure generated by rotating sources. We remark once more that, unlike the spherically symmetric case [1-3], this extension is not based on (quasi-)local quantities, but rather on the asymptotic mass and angular momentum of the Kerr class of space-times. As long as we have no access to local measurements on black hole space-times, this limitation should not be too constraining.

In order to test the capabilities of the so extended HQM, one needs a specific (workable) quantum model of rotating black holes. For this purpose, we have considered the harmonic model for corpuscular black holes [10], which is simple enough to allow for analytic investigations. Working in this framework, we have been able to design specific configurations of harmonic black holes with angular momentum and confirm that they are indeed black holes according to the HQM. Some other results appeared, somewhat unexpected. For instance, whereas it is reasonable that the probability of realizing the inner horizon be smaller than the analogous 
probability for the outer horizon, it is intriguing that the former can indeed be negligible for cases when the latter is close to one. It is similarly intriguing that (macroscopic) extremal configurations do not seem very easy to achieve with harmonic states.

The results presented in this work are overall suggestive of interesting future developments and demand considering more realistic models for self-gravitating sources and black holes. For example, it would be quite natural to apply the HQM to regular configurations of the kinds reviewed in Refs. [23-25].

Acknowledgements R. C. and A. G. are partially supported by the INFN grant FLAG. The work of A. G. has also been carried out in the framework of the activities of the National Group of Mathematical Physics (GNFM, INdAM). O. M. was supported by the Grant LAPLAS 4.

Open Access This article is distributed under the terms of the Creative Commons Attribution 4.0 International License (http://creativecomm ons.org/licenses/by/4.0/), which permits unrestricted use, distribution, and reproduction in any medium, provided you give appropriate credit to the original author(s) and the source, provide a link to the Creative Commons license, and indicate if changes were made.

Funded by SCOAP ${ }^{3}$.

\section{References}

1. R. Casadio, Localised particles and fuzzy horizons: a tool for probing quantum black holes (2013). arXiv:1305.3195 [gr-qc]

2. R. Casadio, What is the Schwarzschild radius of a quantum mechanical particle? in 1st Karl Schwarzschild Meeting on Gravitational Physics, vol. 170, ed. by P. Nicolini, M. Kaminski, J. Mureika, M. Bleicher. Springer Proceedings in Physics (Springer, Switzerland, 2016), p. 225

3. R. Casadio, A. Giugno, A. Giusti, Gen. Relat. Gravit. 49, 32 (2017). arXiv:1605.06617 [gr-qc]
4. R. Casadio, F. Scardigli, Eur. Phys. J. C 74, 2685 (2014). arXiv:1306.5298 [gr-qc]

5. R. Casadio, O. Micu, F. Scardigli, Phys. Lett. B 732, 105 (2014). arXiv: 1311.5698 [hep-th]

6. R. Casadio, O. Micu, D. Stojkovic, JHEP 1505, 096 (2015). arXiv: 1503.01888 [gr-qc]

7. R. Casadio, O. Micu, D. Stojkovic, Phys. Lett. B 747, 68 (2015). arXiv: 1503.02858 [gr-qc]

8. R. Casadio, A. Giugno, O. Micu, Int. J. Mod. Phys. D 25, 1630006 (2016). arXiv:1512.04071 [hep-th]

9. L.B. Szabados, Living Rev. Relat. 12, 4 (2009)

10. R. Casadio, A. Orlandi, JHEP 1308, 025 (2013). arXiv: 1302.7138 [hep-th]

11. W. Mück, G. Pozzo, JHEP 1405, 128 (2014). arXiv:1403.1422 [hep-th]

12. G. Dvali, C. Gomez, JCAP 01, 023 (2014). arXiv:1312.4795 [hepth]

13. G. Dvali, C. Gomez, Black hole's information group (2013). arXiv: 1307.7630

14. G. Dvali, C. Gomez, Eur. Phys. J. C 74, 2752 (2014). arXiv:1207.4059 [hep-th]

15. G. Dvali, C. Gomez, Phys. Lett. B 719, 419 (2013). arXiv:1203.6575 [hep-th]

16. G. Dvali, C. Gomez, Phys. Lett. B 716, 240 (2012). arXiv:1203.3372 [hep-th]

17. G. Dvali, C. Gomez, Fortsch. Phys. 61, 742 (2013). arXiv:1112.3359 [hep-th]

18. G. Dvali, C. Gomez, S. Mukhanov, Black hole masses are quantized (2011). arXiv:1106.5894 [hep-ph]

19. R. Casadio, A. Giugno, O. Micu, A. Orlandi, Entropy 17, 6893 (2015). arXiv:1511.01279 [gr-qc]

20. R.L. Arnowitt, S. Deser, C.W. Misner, Phys. Rev. 116, 1322 (1959)

21. K.S. Thorne, Nonspherical gravitational collapse: a short review. in Magic Without Magic, ed. by J.R. Klauder (San Francisco, 1972), p. 231

22. R. Casadio, A. Giugno, A. Giusti, Phys. Lett. B 763, 337 (2016). arXiv: 1606.04744 [hep-th]

23. P. Nicolini, Int. J. Mod. Phys. A 24, 1229 (2009). arXiv:0807.1939 [hep-th]

24. V.P. Frolov, Phys. Rev. D 94, 104056 (2016). arXiv:1609.01758 [gr-qc]

25. E. Spallucci, A. Smailagic, Regular black holes from semi-classical down to Planckian size (2017). arXiv:1701.04592 [hep-th] 